\title{
Brainstem auditory evoked potential findings in a French bulldog with bilaterally congenital sensorineural deafness
}

\author{
Daegi An ${ }^{1}$, Dong-In Jung ${ }^{2}$, Ha-Jung Kim ${ }^{3}$, Ji-Houn Kang ${ }^{1}$, Dong-Woo Chang ${ }^{1}$, \\ Mhan-Pyo Yang', Byeong-Teck Kang ${ }^{1, *}$ \\ ${ }^{1}$ Veterinary Teaching Hospital, College of Veterinary Medicine, Chungbuk National University, Cheongju 361-763, Korea \\ ${ }^{2}$ Research Institute of Life Sciences, Gyeongsang National University, Jinju 660-701, Korea \\ ${ }^{3}$ Department of Small Animal Clinical Sciences, College of Veterinary Medicine, University of Florida, Gainesville, FL 32615, USA
}

(Received: March 24, 2013; Accepted: September 24, 2013)

\begin{abstract}
A 3-month-old, intact male French bulldog was suspected of deafness. The dog was irresponsive to environmental noises generated out of sight, but normal responses were noted for visual stimuli. No abnormalities were observed on the neurological, otoscopic, radiographic, and blood examinations. To diagnose the apparent deafness, brainstem auditory evoked potential (BAEP) was recorded in the presented dog together with a normal dog. While the BAEP from the control dog showed a normal wave consisting of 5 peaks, absence of all peaks was noted in the suspected deaf dog. Therefore the dog was definitively diagnosed as bilaterally congenital sensorineural deafness.
\end{abstract}

Keywords : brainstem auditory evoked potential, congenital sensorineural deafness, French bulldog

In recent decades, the occurrence of congenital deafness has been increased in dogs relative to the increased awareness among breeders, owners and clinicians [13]. Generally, auditory function is necessary for avoiding traffic accident and other danger incidence. Because deaf dogs are easily surprised and have an increased tendency to bite, they are hardly suitable for use as working. Therefore the puppy with bilateral deafness needs to be trained by a responsible owner, but this kind of training is difficult and unfamiliar. By these reasons, bilaterally deaf puppies are easily euthanized [7].

If the proper diagnosis of hearing impairment could be done in dogs, especially working breeds, it will decrease cost and efforts for being needed in training. Bilateral deafness could be evaluated by monitoring behavioral responses for sound stimuli presented outside of the visual field or with the animal blindfolded, taking care to avoid visual or vibratory cues [11]. However, behavioral testing is often unreliable and subjective [1]. Therefore several objective methods, including electroaudiomerty-encephalographic, audiometry, respiratory audiometry, tympanometry, auditory evoked responses, and acoustic reflexes, have been used for diagnosing audiological or otological disorders in veterinary medicine [10]. Among those methods, the brainstem auditory evoked potential (BAEP) has been proven to be the most objective and non-invasive assessment of auditory function $[14,15]$. Therefore the BAEP is frequently used as a screening test for deafness. Previously congenital deafness had been recognized in
French bulldog, but the BAEP finding has not been reported in this breed [11, 13]. Therefore this report firstly describes the clinical and diagnostic features of bilaterally congenital sensorineural deafness (CSD) in a French bulldog using the BAEP.

A 3-month-old, intact male French bulldog was presented with suspected deafness. This dog was the youngest one from 4 literamates, and the other 3 littermates had no hearing disabilities or other diseases. The owner had not observed any response of the dog for sound stimuli since 1 month ago adoption. There was no history of exposure to any causes of deafness; illness, head trauma or ototoxic drugs. Physical examination revealed mild erythema on the bilateral ear pinna and epidermal collarettes on the abdomen. Its coat color was predominantly white and black, especially white areas presented on the ventral thorax, abdomen, feet, foreleg, and head. On behavior testing, the dog was irresponsive to environmental noises generated out of sight, but normal responses were noted for visual stimuli. Any startle responses were not elicited to sudden loud noises. Bilaterally, intact tympanic membranes and the grossly normal external ear canals were observed on the otoscopic examination. The tympanic bulla was examined by radiography with different views of the open mouth dorsolateral, lateral-oblique, and rostrocaudal projections. Those radiographic studies revealed normal bone density and no fluid density or diminution of foraminal detail within the tympanic bulla. In addition,

*Corresponding author

Tel: +82-43-261-3744, Fax: +82-43-267-2595

E-mail: kangbt@cbu.ac.kr 
A

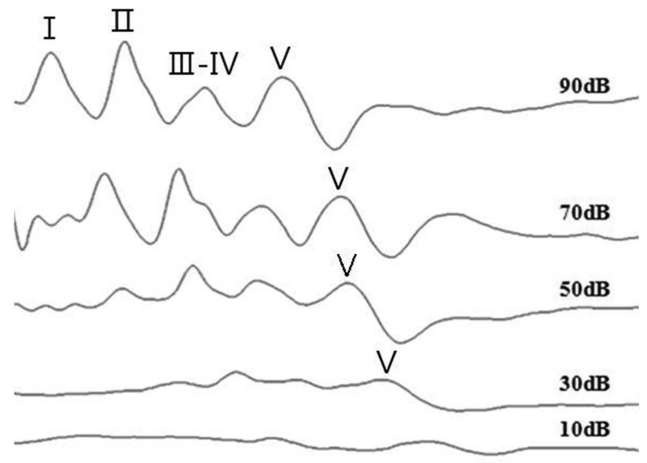

B

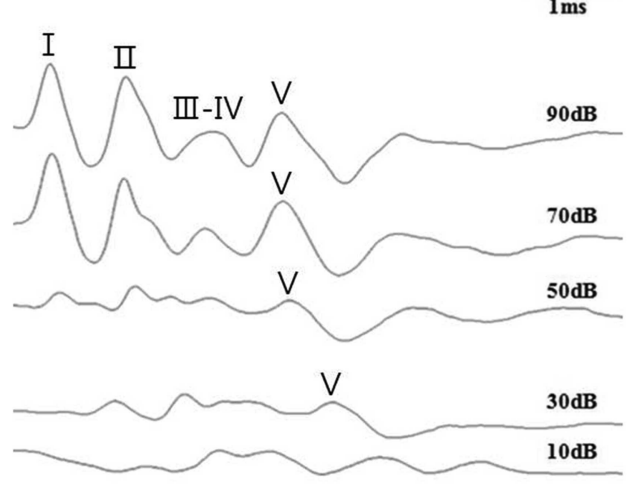

Fig. 1. The brainstem auditory evoked potential (BAEP) recordings in the left (A) and the right (B) ears of a normal dog stimulated by click sound with $90,70,50,30$, and $10 \mathrm{~dB}$ of the normal hearing level. The wave consisting of 5 peaks (I-V) had decreased amplitude and prolonged latency as stimulus intensity decreased. Vertical bar: amplitude, horizontal bar: latency.

abnormalities on the neurological examination, complete blood count, and blood chemistry panel were not remarkable.

Auditory function was objectively assessed by analysis of the BAEP. To prove the validity of the test, the BAEP was also assessed in an adult Maltese dog without hearing problem. After sedating the dogs with medetomidine hydrochloride $(30 \mu \mathrm{g} / \mathrm{kg}$, IM; Domitor; Pfizer, USA) to ensure acceptable recordings by minimizing artifacts generated by muscle movements, they were positioned in sternal recumbency on a padded table in a sound-attenuated room. To detect the BAEP, 10-mm stainless steel needle electrodes (Scalp needle; Natus, Denmark) were placed subdermally on the dogs with the reference electrode at the vertex, the ground electrode over the occiput, and the recording electrode just rostral to the tragus of the ear. After testing the BAEP on the left ear, the recording electrode was moved to the corresponding position on the right ear.

The recording of BAEP was proceeded using an electrodiagnostic machine (Keynote portable; Natus), which was connected with a headphone (Head set; Natus). The click sound (stimulus) with $0.1 \mathrm{msec}$ square wave duration, at a rate of 21.1 clicks/sec, was delivered to the external ear canal by the headphone. The BAEP was recorded for each ear starting at 90 to $10 \mathrm{~dB}$ of the normal hearing level (nHL), decreasing in $20 \mathrm{dBnHL}$ steps. The recordings were obtained as follows: 1) amplifier sensitivity: $5 \mu \mathrm{V} /$ division, 2) sweep speed: $1 \mathrm{msec} /$ division, and 3) band-pass filters: $100 \mathrm{~Hz}$ to $3 \mathrm{kHz}$. The sound stimulus was 1,000 times repeated and averaged.

The BAEP of both ears from the control dog showed the wave consisting of 5 peaks (I-V) with prolonged latency and decreased amplitude as stimuli intensity decreased, however the shape was similar regardless of the intensity of sound stimuli (Fig. 1). Therefore the hearing activity of the control dog was objectively confirmed by identifying normal neuroelectrical activity at various points along the auditory path- way from the inner ear to the level of mid brainstem. On the other hand, all peaks were absent in the BAEP from the both ears of the presented dog with suspected deaf (Fig. 2). This absence of a response reflected profound loss of auditory function in the both inner ears, rather than at several nuclei of the brainstem auditory path, thus confirming bilaterally CSD.

Deafness can be classified as two categories; Central and Peripheral [11]. Theoretically, central deafness can be caused by a variety of retrocochlear lesions, but it is not common in veterinary clinics. Meanwhile, peripheral deafness usually results from the abnormalities of the outside central nervous system. It is usually characterized as inherited or acquired, congenital or later-onset, and sensorineural or conductive [11]. Among various forms of deafness, CSD is the most common type. While acquired conductive deafness can be evoked by otitis externa and/or media, ototoxicity, and noise trauma or presbycusis in older dogs [12], CSD is closely related with pigmentation genes responsible for white in the coat, such as piebald (S), merle (M), and white (W) genes [13]. Strong expression of those genes leads to absent melanocytes in the stria vascularis of the cochlea, which results in pigment-associated deafness through early postnatal degeneration of the stria and secondary degeneration of the cochlear hair cells and neurons [13]. In the present case, the dog had a piebald coloration composed of white and black.

At least 54 breeds of dogs predispose to CSD; especially risk is high in Boston terrier, Dalmatian, English setter, Catahoula leopard dog, Australian cattle dog, Jack Russell terrier, and English cocker spaniel $[11,13]$. The $\mathrm{M}$ gene is seen in the Collie, Shetland Sheepdog, Dappled Dachshund, Harlequin Great Dane, American Foxhound, Old English Sheepdog, and Norwegian Dunkerhound [13]. The S gene is noted in Bull Terrier, Samoyed, Greyhound, Great Pyrenees, Sealyham Terrier, Beagle, Bulldog, Dalmatian, and English Setter [13]. Therefore these breeds are commonly associated with 
A

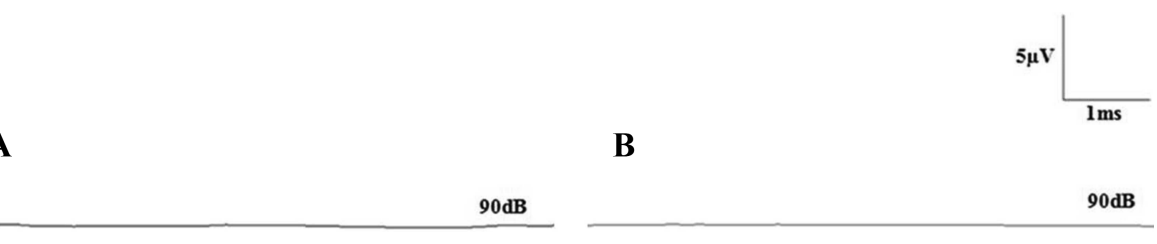

Fig. 2. The brainstem auditory evoked potential (BAEP) recordings in the left (A) and the right (B) ears of a suspected deaf dog stimulated by click sound with $90 \mathrm{~dB}$ of the normal hearing level. The BAEP appeared as a flat line in the both ears in response to sound stimuli. Vertical bar: amplitude, horizontal bar: latency.

CSD. Previously CSD of White Bull Terrier was reported in Korea [5]. In that case, the dog had white coat with a brindle patch on the nose and the final diagnosis was made by the BAEP.

The BAEP is not significantly affected by anesthesia $[2,8$, $9,11,12]$, therefore the present dog was sedated by medetomidine hydrochloride. This drug has been commonly used in dogs and horses during the BAEP procedure [1, 4, 5].

Because of the effectiveness and objectivity, the BAEP test has been commonly used in evaluating the auditory function of animals and humans [3]. The BAEP is usually generated from the vestibulocochlear nerve and auditory portion of the brainstem in response to repetitive sensory stimuli coming from headphone, which is positioned over the dog's ear [15]. The BAEP obtained from animals with normal hearing ability usually consists of 5 to 7 peaks that occur within $10 \mathrm{msec}$ of the stimulus. These peaks reflect the activity of auditory nerve and sequential contributions from several nuclei of the brainstem auditory path [4]. The BAEP data is interpreted by the presence of the expected peaks, latency, and amplitude [8]. If conductive problem can be ruled out, an abnormal BAEP means that the patient has a sensorineural dysfunction. Most investigators report that the BAEP waveforms can be detected in dogs from aged 2 weeks [8], but the cochlear receptor-cell development is continued by the age of 6 weeks [6]. Therefore hearing loss of dogs over this age can be considered to true CSD [6]. In this case, the dog was 3-monthold and no peaks was observed on the BAEP test. In addition, conductive problem could be ruled out by no remarkable abnormalities on the otoscopy and radiography. Therefore the presented dog was definitively diagnosed as bilaterally CSD.

This report describes the clinical and diagnostic features of bilaterally CSD in a French bull dog. The BAEP was a reliable and relatively non-invasive technique available for the evaluation of hearing ability in the dog with suspected deafness.

\section{References}

1. Ahlström LA, Wilson WJ, Mills PC. Unilateral deafness in a white Bull Terrier diagnosed by BAER assessment.
Aust Vet J 2005, 83, 742-743.

2. Cox C. Investigation of hearing loss in dogs. In Pract 2002, 24, 494-501.

3. Harada T, Tokuriki M. Brain-stem auditory evoked potentials the common marmoset (Callithrix jacchus). Electroencephalogr Clin Neurophysiol 1997, 104, 43-50.

4. Harland MM, Stewart AJ, Marshall AE, Belknap EB. Diagnosis of deafness in a horse by brainstem auditory evoked potential. Can Vet J 2006, 47, 151-154.

5. Kang BT, Lee SY, Jung DI, Kim HJ, Woo EJ, Park HM. Bilateral Congenital deafness in a white Bull Terrier: brainstem auditory evoked response findings. J Vet Clin 2008, 25, 506-509.

6. Platt S, Freeman J, di Stefani A, Wieczorek L, Henley W. Prevalence of unilateral and bilateral deafness in border collies and association with phenotype. J Vet Intern Med 2006, 20, 1355-1362.

7. Rak SG, Distl O. Congenital sensorineural deafness in dogs: a molecular genetic approach toward unravelling the responsible genes. Vet J 2005, 169, 188-196.

8. Sims MH. Electrodiagnostic evaluation of auditory function. Vet Clin North Am Small Anim Pract 1988, 18, 913-944.

9. Sims MH, Moore RE. Auditory-evoked response in the clinically normal dog: early latency components. Am J Vet Res 1984, 45, 2019-2027.

10. Sims MH, Shull-Selcer E. Electrodiagnostic evaluation of deafness in two English setter littermates. J Am Vet Med Assoc 1985, 187, 398-404.

11. Strain GM. Aetiology, prevalence and diagnosis of deafness in dogs and cats. Br Vet J 1996, 152, 17-36.

12. Strain GM. Congenital deafness and its recognition. Vet Clin North Am Small Anim Pract 1999, 29, 895-907.

13. Strain GM. Deafness prevalence and pigmentation and gender associations in $\operatorname{dog}$ breeds at risk. Vet $\mathrm{J}$ 2004, 167, 23-32.

14. Strain GM, Kearney MT, Gignac IJ, Levesque DC, Nelson HJ, Tedford BL, Remsen LG. Brainstem auditoryevoked potential assessment of congenital deafness in Dalmatians: associations with phenotypic markers. J Vet Intern Med 1992, 6, 175-182.

15. Wilson WJ, Mills PC. Brainstem auditory-evoked response in dogs. Am J Vet Res 2005, 66, 2177-2187. 